\title{
THE PERFORMANCE OF THE SPH METHOD IN SIMULATING SURFACE RUNOFF ALONG A SATURATED SOIL SLOPE
}

\author{
L.M. DAKSSA \& I.S.H. HARAHAP \\ Civil Engineering Department, Universiti Teknologi PETRONAS, Malaysia.
}

\begin{abstract}
Rainfall-induced slope failures are one of the most disastrous and frequently occurring natural hazards. Hence, it is indispensible to predict their occurrence and their post-failure velocity in order to save lives and properties in mountainous areas. The rain that falls on a soil slope results in either infiltration or surface runoff, depending on the site characteristics. For saturated soil slopes, the amount of rain that goes as infiltration is usually less than the amount that goes as runoff. As a result, surface runoff scours the slope surface, thereby removing the soil slope protecting covers and eventually putting the slope, at least, in a marginally stable condition. This article reports the performance of the smoothed particle hydrodynamics numerical scheme in simulating runoff along a saturated soil slope with emphasis on predicting the velocity of flow. The average velocity of flow using the smoothed particle hydrodynamics method was compared with the average value obtained using a standard open-channel hydraulics empirical equation. The results show that the smoothed particle hydrodynamics method could be used as an alternative method for predicting the runoff velocity along a soil slope in hilly areas.
\end{abstract}

Keywords: Compressible fluid, incompressible fluid, infiltration, meshless numerical methods, runoff, $\mathrm{SPH}$.

\section{INTRODUCTION}

Water is an elixir of life, and its main source is precipitation in the form of rain, snow, mist, hail, etc. Rainwater, as it has unimaginable benefits to ensure the existence of life on Earth, at times, may also disrupt the smooth functioning of the ecosystem. One such detrimental effect is its enormous potential to induce landslides. As a result, soil slope failure triggered by rainfall is reported to be one of the most frequent and dangerous natural hazards (see [1, 2]).

According to Petley (as cited in [3]), in the year 2007 alone, there were 695 landslideinduced fatalities in China, followed by 465 in Indonesia, 352 in India, 168 in Nepal, 150 in Bangladesh, and 130 in Vietnam. In a separate study, Lamay et al. (as cited in [4]) reported the landslide-induced fatality case in Philippines in which a school was buried in 2006 with its 246 students and 7 teachers. In the continental Africa too, for instance, in Ethiopian highlands, the landslide calamity did claim many lives and destroyed properties worth millions of monetary value during the years 1998-2003 alone [5]. On the global context, the Americas (North, Central, and South) and China have the highest number of fatalities [3].

Unfortunately, the life loss and property destructions continue unabated to this date in almost every part of the globe, probably because of the increased exposure of population to the landslide hazard [3]. As the world population keeps on increasing, it puts pressure on governments all around the globe to develop hilly areas. In the prevalence of relatively heavy rainfall events, such actions could lead to the occurrence of devastating landslide hazards.

Rainfall may result in infiltration, runoff, or both depending on the site conditions. Although the infiltrating rainwater reduces the matric suction of the soil slope, runoff scours and, possibly, removes soil slope protecting covers. Once the soil slope covers are removed, 
it is likely that the stability of the soil slope will be compromised. Moreover, for saturated soil slopes the amount of rain that goes as surface runoff is far greater than the amount of rain infiltrating the slope, because soil infiltration rate reduces with soil saturation.

Hence, developmental activities in mountainous areas need to be properly planned to avoid the consequent life losses and injuries. Central to the planning is our understanding of how slope instability is triggered. In this regard, our knowledge pertaining to site geology and geotechnical ameliorative measures is vitally important.

In this article, attempts have been made to investigate the performance of smoothed particle hydrodynamics (SPH) method in capturing some flow characteristics of runoff along a saturated soil slope. As the flow velocity is of paramount importance in planning evacuation strategies, more emphasis has been placed on predicting the runoff velocity.

\section{THE SPH METHODOLOGY}

The smoothed particle hydrodynamics is a numerical approach initially developed for simulating astrophysical phenomena, in 1977 [6] and, later, its applications were reported in different areas of research, including free surface flows, flow through porous media, etc. The SPH meshless numerical method formulation is based on interpolation theory, and two essential concepts dictate its formulation: (i) kernel approximation and (ii) particle approximation.

\subsection{Kernel approximation}

\subsubsection{Kernel approximation of field functions}

Kernel approximation of field functions is, in essence, the representation of the field function(s) in integral form. This is achieved by multiplying an arbitrary field functions with a smoothing kernel function. Therefore, a function $A$ of a three-dimensional position vector $\boldsymbol{x}$ [or an estimate of the function $A(\boldsymbol{x})$ at location $\boldsymbol{x}^{\prime}$ ] can be expressed in integral form as

$$
A(x)=\int_{\Omega} A\left(x^{\prime}\right) \delta\left(x-x^{\prime}\right) d x^{\prime}
$$

where $\delta\left(x-x^{\prime}\right)$ is the Dirac delta function and is given by

$$
\delta\left(x-x^{\prime}\right)= \begin{cases}1 & \text { for } x=x^{\prime} \\ 0 & \text { elsewhere }\end{cases}
$$

where $\Omega$ is the volume of the integral containing $x$ and $x^{\prime}$ is a new independent variable.

In the above expression, function $A(\boldsymbol{x})$ is exact or rigorous, as long as the Dirac delta function is used, and $A(x)$ is continuous in $\Omega$. In SPH, however, the Dirac delta function needs to be replaced by the smoothing (weighting) function $W\left(x-x^{\prime}, h\right)$ in which case it will become an approximate representation of $A(\boldsymbol{x})$. Therefore, the SPH form of a function approximation (or kernel approximation) is expressed as

$$
A(x)=\int_{\Omega} A\left(x^{\prime}\right) W\left(x-x^{\prime}, h\right) d x^{\prime}
$$

where $W$ is called the kernel or a smoothing function and $h$ is the smoothing length, which demarcates the influence area of the smoothing function. However, it needs to be noted that eqn (3) gives an approximate representation of the integral of a field function as long as $W$ is not a Dirac delta function, thus the name kernel approximation. 
2.1.2 The Kernel approximation of a derivative of a function

As equations of computational fluid dynamics problems are mostly partial differential equations (PDEs) of second degree [6], an appropriate approximation of the function derivatives is of profound importance. Accordingly, the kernel approximation of the divergence of the field function $A(\boldsymbol{x})$ (for vector quantity) is therefore expressed as

$$
\nabla \cdot A(x)=\int_{\Omega} \nabla \cdot A\left(x^{\prime}\right) W\left(x-x^{\prime}, h\right) d x^{\prime}
$$

After the application of the divergence theorem, it is always the case where the divergence operation on the primed coordinate in eqn (4) is transferred to the gradient of the smoothing function in the SPH numerical approach, which entails re-writing eqn (4) as

$$
\nabla \cdot A(x)=-\int_{\Omega} A\left(x^{\prime}\right) \cdot \nabla W\left(x-x^{\prime}, h\right) d x^{\prime}
$$

Note that dot product is used in eqn (5). Similarly, the gradient of the function (for scalar quantity) is expressed as

$$
\nabla A(x)=-\int_{\Omega} A\left(x^{\prime}\right) \nabla W\left(x-x^{\prime}, h\right) d x^{\prime}
$$

Therefore, it can be said that the spatial derivative of a field function can be evaluated using the values of the field function and the spatial derivative of the smoothing function. It should also be noted that the negative sign outside the integral sign in eqns (5) and (6) can be removed if the spatial derivative of the kernel function is taken with respect to $\mathbf{x}$ instead of the primed $\mathbf{x}$ (i.e. $\left.x^{\prime}\right)$.

\subsection{Particle approximation}

\subsubsection{Particle approximation of a field function}

Particle approximation is another key operation in the SPH numerical formulation, and it is the means of transforming the continuous kernel approximation (in integral form) into the summation over all particles at the discrete points in the support domain. Particles carry mass, $m$, velocity, $\boldsymbol{v}$, and other quantities specific to the given problem and are regarded as interpolation points, analogous to the grid nodes in mesh-based numerical methods. Therefore, equations that govern the evolution of fluid quantities are expressed as summation interpolants using smoothing function. Equation (9) can then be approximated in a summation form as

$$
A(x) \approx \sum_{j=1}^{N} A\left(x_{j}\right) W\left(x-x_{j}, h\right) \frac{m_{j}}{\rho_{j}}
$$

where $N$ is the total number of particles in the support domain; $m_{j}$ and $\rho_{j}$ are the mass and density of particle $j$, respectively. It should also be noted that the infinitesimal volume $\mathrm{d} \mathbf{x}^{\prime}$ is replaced by the finite volume $\Delta V=m_{j} / \rho_{j}$.

From eqn (7), one can infer that the approximate value of a function at any discrete point can be obtained using the weighted average of those values of the function at all other particles in the influence domain of that particle.

Following a similar argument, the particle approximation for a function at particle (point) $i$ can be expressed as in eqn (8) $[6,7]$ as

$$
A\left(x_{i}\right)=\sum_{j=1}^{N} \frac{m_{j}}{\rho_{j}} A\left(x_{j}\right) W\left(x_{i}-x_{j}, h\right)
$$


2.2.2 Particle approximation of gradient and divergence of field functions

From eqn (8), it can be said that the continuous integral representation of the field function can be expressed in a discretized summation form, which is one of the favourable qualities of the SPH method as that renders the use of a background mesh for numerical integration unnecessarily [5]. Such a conversion of the governing mathematical equations to a workable SPH numerical scheme, however, requires some fundamental techniques as discussed below.

Transformation of the partial differential equations to the SPH discretized summation form, for instance, can be achieved by different methods. One such method is using integration by parts and Taylor series expansion. Suppose that $A$ is a scalar field function representing any physical variable and is defined in a given domain of interest. Then its gradient can be formulated in a manner similar to eqn (8) as follows:

where

$$
(\nabla A)_{i}=\sum_{j=1}^{N} \frac{m_{j}}{\rho_{j}} A_{j} \nabla_{i} W_{i j}
$$

$$
\nabla_{i} W_{i j}=\frac{x_{i}-x_{j}}{\left|x_{i}-x_{j}\right|} \frac{\partial W_{i j}}{\partial r_{i j}}=\frac{x_{i j}}{r_{i j}} \frac{\partial W_{i j}}{\partial r_{i j}}=\nabla_{x i} W_{i j}=-\nabla_{x^{\prime} i} W_{j i}
$$

Applying some basics of vector calculus, other forms of the gradient equations can also be formulated. For instance, substituting $\rho$ inside the gradient operator and applying the chain rule, eqn (9c) can be obtained. The introduction of mass and density into SPH particle approximation is to facilitate numerical approaches in hydrodynamic problems, where density is a key parameter.

$$
\nabla(\rho A)=\rho \nabla A+A \nabla \rho \Rightarrow \nabla A=\frac{1}{\rho}[\nabla(\rho A)-A \nabla \rho]
$$

Thus, re-writing in SPH particle approximation form we obtain

$$
(\nabla A)_{i}=\frac{1}{\rho_{i}}\left(\sum_{j=1}^{N} m_{j}\left(A_{j}-A_{i}\right) \nabla_{i} W_{i j}\right)
$$

The third possible method of approximating the gradient of the field variable $A$ is by incorporating the SPH kernel and particle approximation on a gradient of the unity, which is expressed as follows:

$$
\nabla 1=\int 1 \cdot \nabla W\left(x-x^{\prime}, h\right) d x^{\prime}=\sum_{j=1}^{N} \frac{m_{j}}{\rho_{j}} \nabla_{i} W_{i j}
$$

Obviously, the gradient of the unity is zero. Therefore, eqn $(9 \mathrm{e})$ can be re-written in the form containing both particles $i$ and $j$ as follows:

$$
\nabla 1=\rho_{i} A_{i}\left(\sum_{j=1}^{N} \frac{m_{j}}{\rho_{j}} \nabla_{i} W_{i j}\right)=0
$$

Addition of eqns (9a and 9f) gives another discretized (or discrete) form of the gradient of a field variable and is presented as follows:

$$
(\nabla A)_{i}=\rho_{i} \sum_{j=1}^{N} \frac{m_{j}}{\rho_{j}}\left(A_{i}-A_{j}\right) \nabla_{i} W_{i j}
$$


As it can be seen in eqn $(9 \mathrm{~g})$, unlike in eqn $(9 \mathrm{a})$, the field variable difference is introduced into the discrete particle approximation. Equation $(9 \mathrm{~g})$ is preferred to eqn $(9 \mathrm{a})$, as, in such asymmetric forms, the presence of the variable difference can reduce errors arising from particle inconsistency problems [6]. As a fourth approach, again substituting $(1 / \rho)$ in the gradient operator and applying the chain rule we obtain

$$
\nabla\left(\frac{A}{\rho}\right)=\frac{1}{\rho} \nabla A-\frac{1}{\rho^{2}} A \nabla \rho \Rightarrow \nabla A=\rho\left[\nabla\left(\frac{A}{\rho}\right)+\frac{1}{\rho^{2}} A \nabla \rho\right]
$$

Thus, the SPH equivalent of eqn (9h) is

$$
(\nabla A)_{i}=\rho_{i}\left(\sum_{j=1}^{N} m_{j}\left(\frac{A_{j}}{\rho_{j}^{2}}+\frac{A_{i}}{\rho_{i}^{2}}\right) \nabla_{i} W_{i j}\right)
$$

Finally, if one wishes to develop SPH particle approximation for $\left(\frac{1}{\rho} \nabla A\right)$, it is expressed as

$$
\left(\frac{1}{\rho} \nabla A\right)_{i}=\frac{1}{\rho_{i}}\left(\sum_{j=1}^{N} \frac{m_{j}}{\rho_{j}} A_{j} \nabla_{i} W_{i j}\right)
$$

To obtain a symmetric equation, the concept of a gradient on the unity can be applied here too.

$$
\nabla 1=\frac{A_{i}}{\rho_{i}}\left(\sum_{j=1}^{N} \frac{m_{j}}{\rho_{j}} \nabla_{i} W_{i j}=0\right)
$$

Addition of eqns (9j and $9 \mathrm{k}$ ) yieldsthe following symmetric equation:

$$
\left(\frac{1}{\rho} \nabla A\right)_{i}=\sum_{j=1}^{N} m_{j}\left(\frac{A_{i}+A_{j}}{\rho_{i} \rho_{j}}\right) \nabla_{i} W_{i j}
$$

However, from eqn (9h) we obtain

$$
\frac{1}{\rho} \nabla A=\nabla\left(\frac{A}{\rho}\right)+\frac{1}{\rho 2} A \nabla \rho \Rightarrow\left(\frac{1}{\rho} \nabla A\right)_{i}=\sum_{j=1}^{N} m_{j}\left(\frac{A_{j}}{\rho_{j}^{2}}+\frac{A_{i}}{\rho_{i}{ }^{2}}\right) \nabla_{i} W_{i j}
$$

Therefore, eqns (9a 9d, 9g, 9i, 9j, and 9l) are all correct particle approximations of any scalar field variable $A$ though the symmetric equations are thought to yield accurate results [5]. Following similar procedures as in the case of the gradient of a field variable, the divergence of the field variable can also be expressed in a particle approximation scheme by simply replacing the gradient with the divergence operator.

Note that the negative sign in eqn (5) has been dropped in the above equations, because, here, the spatial derivative of the smoothing function $\nabla W$ is taken with respect to particle $i$ and not with respect to particle $j$.

\subsection{Smoothing functions}

Smoothing (also called weighting) function is at the core of the SPH formulation. Spatial discretization of field variables is based on a set of points (particles, in SPH nomenclature), instead of grid nodes, which are commonly used in mesh-based numerical methods, such as Finite Difference (FD) and Finite Element (FE) methods. Thus, it is with the use of kernel 
interpolation that field variables, such as velocity, pressure, density, stress, etc., are approximated at any point (i.e. at any discrete point) in the support domain. Accordingly, there are several kernel functions being used in the SPH numerical method. The piecewise cubic-spline function, commonly known as B-spline, suggested by Monaghan and Lattanzio (as cited in [6]), is popular among the SPH numerical modelers, such as SPHyscis code developers [8]. The same function is used here as follows:

$$
W(r, h)=\alpha_{d} \begin{cases}\frac{\left(2-\frac{3}{2} q^{2}+\frac{3}{4} q^{3}\right.}{4} & ; 1 \leq q \leq 2 \leq 1 \\ 0 & ; q \geq 2\end{cases}
$$

where $q=\frac{\left|x_{i}-x_{j}\right|}{h}=\frac{r}{h}$ is the relative distance between the two points $x_{i}$ and $x_{j} ;\left|x_{i}-x_{j}\right|=r$ is the distance between the two particles; and, $a_{d}$ is given by $\frac{10}{7 \pi h^{2}}$ and $\frac{1}{\pi h^{3}}$ for two and three dimensions, respectively.

The glaring shortcoming of spline functions is that their second derivative is a piecewise linear function; therefore, the stability properties can be inferior to those of smoother kernels [6]. This could probably be one of the reasons as to why the spatial first derivative of the cubic-spline smoothing functions is widely used in the emerging literature.

\section{SURFACE RUNOFF MODELLING AND SIMULATION}

\subsection{Governing equation}

Traditionally, the velocity of the surface runoff is predicted using the Manning's empirical equation in urban drainage studies or in almost all open channels. For the present study, the Navier-Stokes (NS) equations, combining the mass and momentum conservation equations with the conditions for incompressible fluids, are chosen. As the SPH is the method for compressible fluids in astrophysical studies, its application to incompressible fluids is under the assumption that every fluid could be treated as compressible to $<1 \%$ [6]. Meaning, density variations of $1 \%$ could be allowed without affecting the solutions significantly.

As a result, the NS equation has been widely used to model incompressible flows through porous media and/or free surface flows by several researchers, e.g. [9-12]. The NS equation models flow at the micro or pore-scale level. In [13] the interaction between water waves and porous medium was investigated using what was dubbed the NS-type equation. The NS equations were also used in [14] for simulating free surface flow using SPH, with the exception that the artificial viscosity term was used instead of the laminar viscosity. In a separate study Morris et al. [15] employed the same continuity and momentum equations to model incompressible low Reynolds number flows.

Therefore, the governing equation is given by

$$
\frac{1}{\rho} \frac{D \rho}{D t}+\nabla \cdot V=0
$$


Equation (11) is the mass conservation equation. Again, for momentum conservation (incompressible fluid), we obtain

$$
\frac{D V}{D t}=-\frac{1}{\rho} \nabla P+v \nabla^{2} V+g
$$

where $\boldsymbol{V}$ is the velocity vector, $\rho$ is the water density, $g$ is the gravity, $P$ is the pressure, $v$ is the dynamic viscosity, and $t$ is the time.

It is also worth noting that the SPH was originally invented for modelling flows of compressible fluids, and thus its application to incompressible fluid flows needs some approach to ensure that density variation within a certain limit is maintained. This is discussed in the following section.

\subsection{Equation of state}

For the standard SPH for compressible fluids, pressure gradient is normally solved using equation of state (EoS). However, for the case of incompressible fluids, solving the pressure using Poisson pressure equation dictates the adoption of a small time-step [6]. This constraint has led to the adoption of artificial compressibility for solving the pressure gradient in the NS equations. Accordingly, Monaghan [14] modified the EoS suggested for water by Batchelor (also in [14]), for describing sound waves and used it for simulating free surface flows. In this research too the same EoS is used as given in eqn (13). Moreover, Bui et al. [16] applied the same EoS in their formulation of SPH for soil mechanics with some successes, which is given by

$$
P=B\left[\left(\frac{\rho}{\rho_{o}}\right)^{\gamma}-1\right]
$$

where $\gamma$ is a constant and is taken to be 7 for most circumstances; $\rho_{0}$ is the reference density; and $B$ is a problem-dependent parameter for limiting the maximum density gradient and, in most cases, can be taken as the initial pressure $[6,15]$.

\subsection{Viscous term in the NS equations}

The Laplacian for calculating the viscous term in the fundamental NS equation [see eqn (12)] has been dealt with differently by different researchers. Liu and Liu [6], for instance, employed artificial viscosity. In [15] a new approach was developed, which is quite similar to the expression used in [16]. This article chooses the expression used in [8], which is given as in (14) for calculating the viscous term at particle $i$ and is expressed as

$$
\left(v \nabla^{2} \mathbf{V}\right)_{i}=2 v \sum\left(\frac{r_{i j} \cdot \nabla_{i} W_{i j}}{\bar{\rho}_{i j}\left|r_{i j}\right|^{2}}\right) V_{i j}
$$

where $v$ is the kinematic viscosity; $r_{i j}$ is the distance between the $i$ and $j$ particles; $v_{i j}$ is the velocity difference between particle $i$ and particle $j$; and $W_{i j}$ is the smoothing length.

\subsection{Boundary treatment}

Boundary treatment entails special consideration in SPH, as particle deficiency near or on the boundary impairs full exploitation of the scheme. Monaghan [14], also reported in [6], 
suggested the use of ghost particles near or on the boundaries so that a high repulsive force is created to prevent the fluid particles from unphysically penetrating a solid boundary. Such a penalty force approach to prevent interior fluid particles from penetrating the boundary is based on the Lennard-Jones molecular force approach. Another approach, in which the Hertzian contact theory was used, was also developed in [16]. For the current research, we use the Monaghan approach as given in eqn (14) and is given by

$$
P B_{i j}= \begin{cases}D\left[\left(\frac{r_{o}}{r_{i j}}\right)^{a}-\left(\frac{r_{o}}{r_{i j}}\right)^{b}\right] \frac{x_{i j}}{r_{i j}^{2}} & \left(\frac{r_{o}}{r_{i j}}\right) \leq 1 \\ 0 & \text { elsewhere }\end{cases}
$$

where, as in [6], $a$ and $b$ are taken to be 12 and 4, respectively, although Monaghan [14] proposed 4 and 2, respectively, with the conditions that $a>b$, always. He also suggested that $a$ and $b$ could be taken as 12 and 6 , respectively, without significant changes in the results. $D$ is a problem-dependent parameter and is usually taken to be the square of the largest velocity [6], and $r_{0}$ is selected to be approximately equal to the initial particle spacing.

\subsection{Time integration}

Here, the predictor-corrector method is used. The predictor step predicts a new value, and the corrector step improves the accuracy of that value. The predictor step is undertaken only once for each of the iterations, whereas the corrector step is continued until the required level of accuracy is reached. There are several predictor-corrector methods; although for this article we stick to the Euler predictor-corrector method (some prefer to name it as modified Euler method). The basic idea behind the second-order Euler predictor-corrector method is that the solution for a new time-step is predicted using the explicit Euler method and the final solution is corrected by applying the trapezoid rule as follows:

$$
\begin{gathered}
\varphi^{(n+1)^{*}}=\varphi^{n}+f\left(t_{n}, \varphi^{n}\right) \Delta t \\
\varphi^{(n+1)}=\varphi^{n}+\frac{1}{2}\left[f\left(t_{n}, \varphi^{n}\right)+f\left(t_{n+1}, \varphi^{(n+1)^{*}}\right)\right]
\end{gathered}
$$

For stability, the time-step, $\Delta t$, needs to be checked against several stability requirements. A detailed reading regarding these stability conditions can be made in $[6,15]$.

\section{EXAMPLES AND DISCUSSIONS}

In this section numerical calculations and discussions are made. The data used are as follows: the rainfall intensity (surface-flux) is $10^{-4} \mathrm{~m} / \mathrm{s}$; the slope angle is $-40^{\circ}$; and the kinematic viscosity is $1 \times 10^{-6} \mathrm{~m}^{2} / \mathrm{s}$.

Figure 1 shows the configuration of the slope physical model before and after the onset of surface runoff. Figure 1a depicts the initial slope configuration, the cyan colour representing the soil particles, the red colour representing impenetrable boundary particles, and the blue colour representing water particles. For the current simulation one main program with a number of subroutines was coded to develop the initial configuration. A separate set of program codes were also coded for inducing runoff. Different simulation examples were carried out for different time-steps and simulation times. The left, top, and the bottom boundaries were treated as no-flow boundaries. In the coding, it was possible to record automatically, in a 


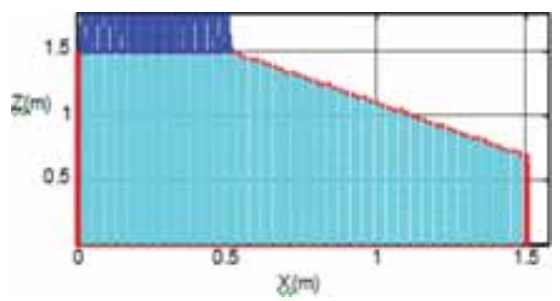

(a)

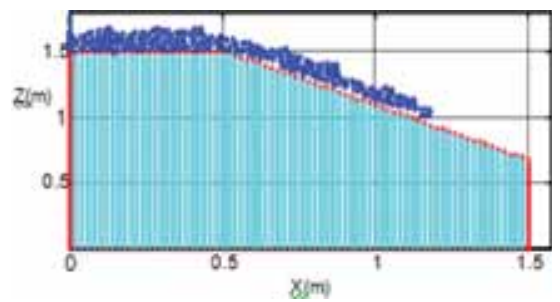

(c)

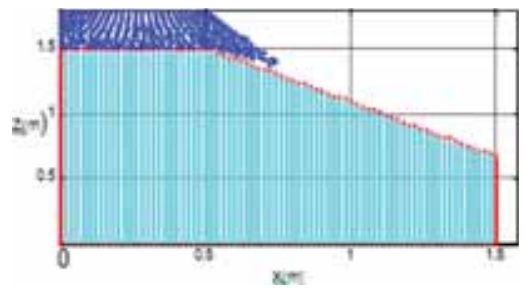

(b)

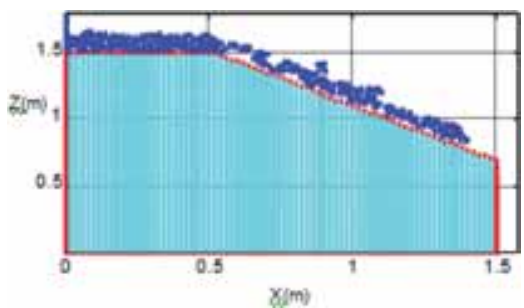

(d)

Figure 1: The SPH simulation for a simulation time of $\sim 0.5 \mathrm{~s}$ and a time-step of $0.02 \mathrm{~s}$.

separate file, the positions of each particle, their respective velocities, densities, and porewater pressures. Those data files were used for the graphics using MATLAB. As can be noted from Fig. 1b-d, the SPH scheme is a suitable means for capturing different aspects of the runoff.

\subsection{The average velocity from open-channel hydraulics}

The Manning's empirical equation is popular in urban drainage projects. It also enjoys broad applications in irrigation water supply projects. Manning's coefficient of channel roughness is the value accounting for flow retardation due to vegetations and other obstructions on the soil slope. The values of $\eta$ play an important role in velocity computation. Accordingly, the minimum and the maximum roughness coefficient values were sought so that the minimum and the maximum velocity values would be calculated. The different open channels along with their respective $\eta$ values in which the minimum $\eta$ value ranges from 0.011 (for a concrete lined channel with trowel finish) to 0.110 (for natural streams/floodplains with dense willows) are listed in [17]. Given the section, taking the average of the two $\eta$ values (i.e. 0.061 ), and plugging it into Manning's equation, the average velocity can be obtained. The average velocity for the current section (a width of $0.5 \mathrm{~m}$ and a water depth of $0.48 \mathrm{~m}$ ) is, therefore, $\sim 4.50 \mathrm{~m} / \mathrm{s}$. This maximum average velocity was compared with the maximum velocity obtained using the SPH simulation for a time-step of $0.02083 \mathrm{~s}$ and is summarized in Table 1.

The average maximum velocity can be calculated as follows:

$$
\boldsymbol{V}^{2}=(2.89)^{2}+(-2.62)^{2}=>\boldsymbol{V} \cong 4.0 \mathrm{~m} / \mathrm{s}
$$

Comparing the two average maximum velocities obtained using the Manning's empirical equation $(4.50 \mathrm{~m} / \mathrm{s})$ and the $\mathrm{SPH}$ approach $(3.90 \mathrm{~m} / \mathrm{s})$, the authors are of the opinion that the $\mathrm{SPH}$ approach reasonably predicted the velocity of the flow. 
Table 1: The maximum velocities during each time-step by the SPH scheme.

\begin{tabular}{lcc}
\hline Iteration & $\begin{array}{c}\text { The maximum velocity in } \\
\text { the } x \text {-direction }(\mathrm{m} / \mathrm{s})\end{array}$ & $\begin{array}{c}\text { The maximum velocity in } \\
\text { the } z \text {-direction }(\mathrm{m} / \mathrm{s})\end{array}$ \\
\hline 1 & 0.74 & 0.19 \\
2 & 1.02 & 0.39 \\
3 & 1.16 & 0.60 \\
4 & 1.23 & 0.81 \\
5 & 1.56 & 1.01 \\
6 & 1.77 & 1.52 \\
7 & 1.96 & 1.35 \\
8 & 1.96 & 1.69 \\
9 & 2.16 & 2.17 \\
10 & 2.75 & 2.00 \\
11 & 2.61 & 2.31 \\
12 & 2.95 & 2.85 \\
13 & 3.35 & 2.72 \\
14 & 3.16 & 3.09 \\
15 & 3.30 & 3.06 \\
16 & 3.28 & 3.24 \\
17 & 3.62 & 3.55 \\
18 & 3.75 & 3.75 \\
19 & 3.53 & 4.44 \\
20 & 3.63 & 4.30 \\
21 & 4.92 & 4.39 \\
22 & 5.33 & 4.06 \\
23 & 4.29 & 4.03 \\
24 & 5.36 & 5.40 \\
Average & 2.89 & 2.62 \\
Maximum Average $V$ & 4.0 & \\
\hline & & \\
& & \\
& &
\end{tabular}

\section{CONCLUSIONS}

In this research, the SPH scheme was employed to simulate surface runoff along a saturated soils slope. The NS equations govern the flow conditions. The simulation outputs include the respective particle velocities, positions, mechanical pressures, and densities, even though only the velocities are presented in this article. The snapshots of the flow are also shown. Acknowledging the need for further improvements, especially, with respect to incorporating the actual fluid rheological properties, this preliminary finding suggests that the SPH scheme can be used as an alternative to or in conjunction with the existing runoff velocity prediction empirical formulas. Predicting the flow velocities is especially required for emergency planning in areas vulnerable to landslide.

\section{REFERENCES}

[1] Ng, W.C.C. \& Shi, Q., A numerical investigation of the stability of unsaturated soil slopes subjected to transient seepage. Computers and Geotechnics, 22(1), pp. 1-28, 1998. doi: http://dx.doi.org/10.1016/S0266-352X(97)00036-0

[2] Tsaparas, I., Rahardjo, H., Toll, D.G. \& Leong, E.C., Controlling parameters for rainfallinduced landslides. Computers and Geotechnics, 29, pp. 1-27, 2002. doi: http://dx.doi. org/10.1016/S0266-352X(01)00019-2 
[3] Kjekstad, O. \& Highland, L., Economic and social impact of landslides. Landslides Disaster Risk Reduction, eds K. Sossa \& P. Canuti, Springer: Berlin and Heidelberg, pp. 573-587, 2009. doi: http://dx.doi.org/10.1007/978-3-540-69970-5 30

[4] Borja, R.I., Landslides and debris flow induced by rainfall. Insights. Institute of Advanced Study, Vol. 2, No. 3, Durham University, 2009.

[5] Abay, A. \& Barbieri, G., Landslide susceptibility and causative factors evaluation of the landslide area of Debresina, in the southwestern Afar escarpments. Ethiopia. Journal of Earth Science and Engineering, 2, pp. 133-144, 2012.

[6] Liu, G.R. \& Liu, M.B., Smoothed particle hydrodynamics - a meshfree particle method, World Scientific Publishing, Singapore, 2003.

[7] Bui, Ha H., Sako, K. \& Fukagawa, R., Numerical simulation of soil-water interaction using smoothed particle hydrodynamics (SPH) method. Journal of Terramechanics, 44, pp. 339-346, 2007. doi: http://dx.doi.org/10.1016/j.jterra.2007.10.003

[8] Gesteira, M.G., Rogers, B.D., Dalrymple, R.A., Crespo, A.J.C. \& Narayanaswamy, M., User Guide for the SPHysics Code v2.0, 2010.

[9] Narsilio, G.A., Buzzi, O., Fityus, S., Yun, T.S. \& Smith, D.W., Upscaling of NavierStokes equations in porous media: theoretical, numerical and experimental approach. Computers and Geotechnics, 36, pp. 1200-1206, 2009. doi: http://dx.doi.org/10.1016/j. compgeo.2009.05.006

[10] Jiang, F. \& Sousa, A.C.M., Smoothed particle hydrodynamics modelling of transverse flow in randomly aligned fibrous porous media. Transport in porous media, $\mathbf{7 5}$, pp. 17-33, 2008. doi: http://dx.doi.org/10.1007/s11242-008-9206-Z

[11] Morris, J.P., Zhu, Y. \& Fox, P.J., Parallel simulations of pore-scale flow through porous media. Computers and Geotechnics, 25, pp. 227-246, 1999. doi: http://dx.doi.org/10.1016/ S0266-352X(99)00026-9

[12] Pereira, G.G., Prakash, M. \& Cleary, P.W., SPH modelling of fluid at the grain level in a porous medium. Applied Mathematical Modelling, 35, pp. 1666-1675, 2011. doi: http://dx.doi.org/10.1016/j.apm.2010.09.043

[13] Shao, S., Incompressible SPH flow model for wave interactions with porous media. Coastal Engineering, 57,pp. 304-316, 2010. doi: http://dx.doi.org/10.1016/j.coastaleng.2009.10.012

[14] Monaghan, J.J., Simulating free surface flows with SPH. Journal of Computational Physics, 110, pp. 399-406, 1994. doi: http://dx.doi.org/10.1006/jcph.1994.1034

[15] Morris, J.P., Fox, P.J. \& Zhu, Y., Modelling low Reynolds number incompressible flows using SPH. Journal of Computational Physics, 136, pp. 214-226, 1997. doi: http:// dx.doi.org/10.1006/jcph.1997.5776

[16] Bui, Ha H., Fukagawa, R. \& Sako, K., Smoothed particle hydrodynamics for soil mechanics. Numerical Methods in Geotechnical Engineering (eds.), Taylor \& Francis, 2006.

[17] Tennessee Department of Transportation (TDOT), design division, drainage manual, roadside ditches and streams, May 15, 2011.

"This is an extended version of the paper published in WIT Transactions on Engineering Sciences, Vol 73, (C) 2012 WIT Press, www.witpress.com, ISSN 1743-3533 (on-line), doi: 10.2495/ DEB120201." 